\title{
Cell biology considerations in Spinal Cord Injury - Review
}

\author{
Constantin Munteanu ${ }^{1}$ \\ 1.Romanian Association of Balneology, Bucharest, Romania, www.bioclima.ro, office@bioclima.ro
}

\begin{abstract}
Spinal cord injury (SCI) affects more than 2,5 million people worldwide, with more than 130000 new injuries reported annually. SCI is the result of an aggression on the spinal cord, which totally or partially compromises its functions (motor, sensory, vegetative, reflex). SCI ends in $15 \%$ of cases with the victim's death before reaching the hospital. Also, a mortality of about. $5 \%$ is registered at the level of specialized assistance centers capable of providing qualified, multidisciplinary assistance, while in non-specialized centers the mortality can be between $25-40 \%$. As soon as a severe bone marrow lesion occurs, the spinal cord enters a state of diminished excitability. This condition is called spinal shock or altered reflex activity. Transient inhibition of caudally located segments of the lesion is due to the sudden disappearance of the predominantly facilitating or excitatory influence of supraspinal centers. Thus there is a flaccid, flaccid paralysis. The duration of the spinal shock varies, a minimal refractive activity may occur within 3-4 days or only after 6-8 weeks, with an average duration of 3-4 weeks, after which occurring sequelae due to the medullary lesion, while medullary neurons under the lesion level becomes autonomous to the influences of the upper floors. The degree of neurological injury and neurological deficit is determined by the extent and severity of the action of these factors. Pain in patients with SCI dresses almost all possible variants from acute pain related to tissue trauma, colic pain caused by the presence of a lithiasis, various types of headache and especially medullary pain. The latter encounter an important proportion of people with a tetra or paraplegia. The pains encountered can be divided into five distinct categories: diffuse pain; segmental pain; root pain; visceral pain; and neurogenic pain. Fever is often present, especially in acute phases. Its value as a symptom of an infection should be carefully analyzed before administering anti-thermic drugs. Urinary, respiratory and esophageal infections are part of the clinical picture of many patients. Consequently, their correct diagnosis and diagnosis followed by the introduction of the treatment are the law. A mention should be made of the so-called fever of undetermined origin, often encountered in early stages, especially in patients with localized cervical medullary lesions. This fever, usually refractory to current treatments, would, in some cases, explain the disturbance of the afferent to the thermoregulation centers. Before declaring the existence of such a fever, it is necessary first to eliminate other causal factors. The balancing recovery step comprises the application for prophylactic and curative purposes of a variety of procedures based on water as a natural therapeutic factor at different temperatures and different states of aggregation as well as specific techniques.
\end{abstract}

Key word: Spinal Cord Injury (SCI), seccondary medullary lesions, neuroplasticity, apoptosis 


\section{Introduction}

The pathophysiology of acute spinal cord injury (SCI) involves primary and secondary mechanisms of injury, both involved in the neurological dysfunction in SCI [1].

The primary lesion is the mechanical injury itself, and the secondary lesion results from one or more biochemical and cellular processes that are triggered by the primary lesion [4].

\section{Spinal Cord Anatomy}

In order to better understand the complex pathophysiological changes that occur following SCI, it is important to first understand the anatomy and function of the spinal cord region in a healthy individual. The spinal region is made up of neural structures housed within the vertebrae including the spinal cord, dorsal and ventral roots, spinal nerves, and meninges. The spinal cord begins from the medulla oblongata, just above the C1 vertebral level and ends at the L1-L2 intervertebral space. Inferior to this level, long lumbar and sacral nerve roots extend to form the cauda equina.

The internal anatomy of the spinal cord is divided into white and gray matter. The white matter contains the axons of both sensory and motor neurons that are ascending and descending along the spinal cord. The white matter is further divided into dorsal, lateral, and anterior columns depending on the type of information being carried in the fibers running through each region. The central part of the cord is made of gray matter which houses the terminal synapses of axons from other areas, neuronal cell bodies and dendrites, and glial cells (nonneuronal cells that help with spinal cord function and survival). The gray matter is divided into dorsal, lateral, and ventral horns. The dorsal horn contains mostly sensory neurons, the lateral horn (only present from T1-L1) contains the cell bodies of neurons involved with the sympathetic visceral innervation (help with autonomic regulation), and the ventral horn contains cell bodies of lower motor neurons that innervate skeletal muscle.

\section{Primary lesion in SCI}

A primary lesion that is caused by an impact to the spinal cord consists of acute structural and physiological disruption of axons, nerve cell damage and blood vesel ruptures. Hemorage and necrosis in the central gray matteroccur within the first hours after the injury (acute phase), followed by edema and hemorrhage in the seven hours following the trauma. The injury is the result of ischemia that is caused by reduced blood flow to the affected spinal segment. This reduction may be caused by a change in the spinal canal, by significant edema and hemorrhage or by reduced systemic blood presure [4].

There are four main characteristic mechanisms of primary injury which include:

- Impact plus persistent compression

- Impact alone with transient compression

- Acute distraction of the spinal cord

- Laceration or transection of the spinal cord The most common mechanism of injury is the impact plus persistent compression, which can be seen following a burst fracture of the spine, a fracture-dislocation of the spine, or an acute disc rupture into the spinal cord. Impact along with transient compression is often seen with hyperextension injuries in patients with an underlying degenerative spine disease. Acute distraction of the spinal cord can occur following extreme flexion, extension, or rotation that causes shearing or stretching of the spinal cord. Lacerations can occur from violent injuries or sharp bone fragment dislocations and vary from minor to complete transections [6,7].

\section{Secondary lesion in SCI}

In SCI, the primary mechanical damage is followed by a complex process of secondary damage due to inflammation, ischemia, free radical production, and apoptosis, among other processes. Massive disruption of the descending and ascending axonal networks, loss of neuronal and glial cells, myelin damage, and the formation of cysts and glial scars characterize the patophysiology of the injuried spinal cord. After SCI, many severed axons at the lesion site sprout at their cut ends, but than retract because central nervous 
system (CNS) axons have a poor intrinsic capacity for growth and encounter a series of inhibitory factors that are nonpermissive for growth such as myelin inhibitors (Nogo-A), myelin-associated glycoprotein (MAG), and oligodendrocyte myelin glycoprotein (Omgp), as well as proteoglycans, such as chondroitin sulphate proteoglicans, which contribute to glial scar[2].

Ischemia creates a chain of biochemical reactions that result in cell death. Inflammatory cells then simultaneously migrate to the injured site with glial cell proliferation. The chronic phase lasts one to four weeks; during this time, the proliferation and hypertrophy of astrocytes from a glial scar or a cyst [4].

Functional deficites following SCI result from damage to or severance of axons, loss of neurons and glia, and demyelination. SCI pathology is determined not only by the initial mechanical insult, but also by secondary processes including ischemia, anoxia, free radical formation, and excitotoxicity that occur over hours and days following injry. Central nervous system (CNS) axonal regeneration appears to be impeded partly by myelinassociated inhibitors, loss in adult neurons of an intrinsic ability to overcome inhibitory cues, and formation of a post-lesion scar barrier. However, if axons can traverse the injury site, there is evidence that they may regrow in unscarred regions. Furthermore, preservation of even a small percentage of tissue significantly enhances functional recovery [5].

Secondary injury is a result of the progressive destruction from the primary injury that further exacerbates the injury to the cord itself. The main mechanisms which create the overall effects on the spinal cord are often associated with vascular changes, free radicals, excitotoxicity, and cell death.

Secondary injury often presents within two hours post-injury, where the damage begins to travel rostrally and caudally from the primary lesion. At this point, edema and the hemorrhaging within the gray matter increase over the next few hours, before damage to the white matter becomes evident $[6,7]$.

\section{Systemic Effects}

Injury to the spinal cord can lead to neurogenic shock and respiratory failure through the disruption of the autonomic nervous system's (ANS) ability to regulate the body. Neurogenic shock is a transient phase that occurs immediately after trauma in which spinal reflexes, voluntary motor and sensory function, and autonomic control are absent or depressed below the lesion. The loss of autonomic regulation leads to the brief increase in heart rate and systemic blood pressure seen post-SCI, as well as the following prolonged bradycardia and hypotension. The loss of tone in blood vessels leads to pooling of blood in the arteries and veins further from the heart causing the prolonged hypotension. These initial changes lead to decreased peripheral vascular resistance, decreased cardiac output, hypoxia, and hyperthermia [6,7].

\section{Local Vascular Effects}

Following the initial traumatic impact, local changes occur to the vascular supply of the spinal cord, ultimately leading to both hemorrhagic and ischemic states. There is an initial reduction of blood flow, and within 15 minutes of the acute injury, hemorrhaging occurs in the gray matter and edema occurs in the surrounding white matter [6,7].

Edema in the cord can cause further compression of the meninges, leading to further disruption of the spinal cord by damaging its protective layers. Vessels in the gray matter become swollen with erythrocytes causing small petechial hemorrhaging in the perivascular space. The hemorrhaging extends rostrally and caudally over the next few hours following the initial insult. Microcirculation, including venules and capillaries, experience more extensive damage than larger vessels.

Ischemia begins to progressively worsen over the first few hours post-SCI, although the mechanisms are still unclear $[6,7]$.

Possible causes include vasospasms which are induced from the initial trauma, intravascular thrombosis formation due to platelet aggregation, and the presence of increased norepinephrine in the blood supply. Ischemia 
can lead to local edema and endothelial swelling and damage of capillaries and venules within 1-2 hours post-SCI. The central gray matter and adjacent white matter are most affected acutely because the ratio of blood flow is approximately 3:1 for gray to white matter. Axonal damage is also noted due to disruption of the myelin sheath. Obstruction of the anterior sulcal arteries and arterioles may lead to hemorrhagic necrosis at the primary injury site [6,7].

Overall the central nervous system is intolerant to ischemia and as little as $15-30$ seconds without oxygen can lead to irreversible damage in neurons. After the initial stage of reduced perfusion, a period of hyperemia occurs due to reduced perivascular $\mathrm{pH}$ from the accumulation of acidic metabolites (e.g. lactate). This reperfusion can actually worsen the injury and increase necrosis because it produces harmful free radicals and other reactive oxygen species.

One of the local vascular effects following SCI is infarction, or cell death due to loss of oxygen. Loss of oxygen in neurons perpetuates necrotic cell death in the CNS tissue through various mechanisms. The initial injury leads to loss of cell membrane integrity, vascular vasospasms, and thrombosis, which ultimately lead to a decrease in oxygen and glucose being provided to the cell. Without sufficient oxygen and glucose, there is a disruption in the processes of glycolysis and oxidative phosphorylation, which are the main cellular cascades used to produce ATP in the cell. With a decrease in ATP, the cells experience disruption in their membrane permeability and changes in ion concentrations, release of lysosomal enzymes which break down the cell, and increases in intracellular calcium which sets of the process of calcium-mediated cell death [6,7].

\section{Excitotoxicity}

Excitotoxicity is defined as neuronal death caused by excessive or prolonged activation of glutamate receptors. In uninjured spinal cords, glutamate is an excitatory neurotransmitter involved with the depolarization of axons of spinal neurons.
When an axon is depolarized, glutamate is released from the nerve terminals, where it then crosses the synaptic cleft to act on postsynaptic ionotropic receptors, including N-methyl-D-aspartate (NMDA), $\alpha$-amino-3hydrox-5-methyl-4-isoxazolepropionic acid (AMPA), and kainate. NMDA is a ligandgated ion channel that permits entry of sodium and calcium ions and efflux of potassium ions. AMPA and kainate are non-NMDA, ligandgated channels that regulate sodium influx and potassium efflux when activated by glutamate. NMDA receptors are involved in secondary glutamatergic synaptic transmission once the neuron has been depolarized by activation of AMPA/kainate receptors. The activation of these ionotropic receptors leads to further depolarization; when a certain threshold is reached an action potential is generated. Normally, glutamate is rapidly removed from the synapse through uptake systems including excitatory amino acid transporters (EAAT). EAAT are proteins found in the membranes of glial cells (including astrocytes, oligodendrocytes, and microglia), neurons, and endothelial cells. They transport glutamate, along with other ions, across cell membranes, and quickly remove it from the extracellular space. This quick removal helps to maintain extracellular levels of glutamate and also stops the synaptic transmission [6,7]. Excitotoxicity occurs as a result of chronic depolarization of neurons caused by increased levels of extracellular glutamate. Changes in sodium $\left(\mathrm{Na}^{+}\right)$and calcium $\left(\mathrm{Ca}^{2+}\right)$ ion influx, as well as exocytosis of glutamate, all play a role in the mechanism of excitotoxicity. Depolarization leads to increased activation of AMPA, and kainate. When glutamate binds to the AMPA or kainate receptors, depolarization occurs, which in turn activates voltagedependent $\mathrm{Na}^{+}$channels, causing even further depolarization and an increase in intracellular $\mathrm{Na}^{+}$. Cells will begin to increase their influx of chloride ions to attempt to balance out the sodium influx. Ultimately, the osmotic balance of the cell is so disrupted that the cell begins to increase its water intake. Cell lysis occurs causing cellular contents, including glutamate, to leak back into extracellular space. 
Additionally, there is increased activation of NMDA receptors, which leads to an increase in intracellular $\mathrm{Ca}^{2+}$.

Calcium ions enter the cell through the activated NMDA channels and further disrupt cellular homeostasis. As more and more cell lysis occurs, glutamate levels increase, there is slowing of the glutamic acid transport chain, and calcium-mediated cell death begins.

Cell death from excitotoxicity also occurs via changes in ion levels, calcium- dependent mechanisms, dysfunctioning mitochondria, and the production of free radicals and other reactive oxygen species (ROS). Increases in $\mathrm{Na}^{+}$influxes triggers an increase in $\mathrm{Ca}^{2+}$ influx through voltage-gated calcium channels, as well as reversal of sodiumcalcium membrane exchangers. High sodium levels also lead to edema and intracellular acidosis. Sodium-potassium ATPase fails during excitotoxicity causing increasing accumulation of $\mathrm{Na}^{+}$, water, and $\mathrm{Ca}^{2+}$ into the cell, and increasing loss of potassium $\left(\mathrm{K}^{+}\right)$into extracellular space [6,7].

\section{Free Radicals and Reactive Oxygen Species}

Glutamate neurotoxicity is also mediated by the generation of free radicals and reactive oxygen species. Free radicals disrupt cellular organelles and initiate cell-programmed death, apoptosis. NMDA receptor-mediated excitotoxicity initiates a cascade of events that ultimately leads to the genesis of reaction molecules that contribute to neuronal death through a variety of mechanisms. Lipid peroxidation can begin that can produce free radicals, as well as an alteration in the integrity of the sodium potassium pumps. Regular mitochondrial functions that produce free radicals can become damaging after SCI.

In the mitochondria, the free radical superoxide $\left(\mathrm{O}^{2-}\right)$ is produced as a by-product of oxdative phosphorylation. $\mathrm{O}^{2-}$ is normally detoxified by superoxide dismutase (SOD) which produces the more stable moledcule of hydrogen peroxide (H2O2). When transition metal ions such as iron are present, the hydrogen peroxide is reduced and a hydroxyl $\left(\mathrm{OH}^{-}\right)$free radical is produced. Luckily, cells have built-in antioxidant systems to keep the levels of free radicals below a toxic threshold. One example is the mitochondrial antioxidant manganese superoxide dismutase (MnSOD) which detoxifies superoxide to produce hydrogen peroxide. Another example is glutathione peroxidase (GPx) that can detoxify hydroxyl free radicals into water. When free radicals are produced faster than they can be detoxified, or if the detoxification systems are impaired, the build-up of reactive oxygen species can lead to damage of mitochondrial proteins, lipids, and nucleic acids. With these impaired, oxidative phosphorylation cannot occur and more and more free radicals will be produced. Eventually this leads to apoptotic cell death. Free radicals have been implicated as harmful contributors to the secondary injury following the initial SCI. Nitric oxide (NO) levels increase following SCI which impairs mitochondrial respiration by binding to respiratory enzyme Complex IV. Superoxide anion (O2-) causes damage by impacting Complex III of the oxidative phosphorylation process $[6,7]$.

\section{Ion Channels \& Concentrations}

\section{Potassium}

Potassium $\left(\mathrm{K}^{+}\right)$channels are located on myelinated axons and facilitate in the production of action potentials in order to stimulate neuronal conduction. They are also essential in cardiac muscle and assist in regulating hormone secretions in the body. There are three types of $\mathrm{K}^{+}$channels: 1 ) Fast $\mathrm{K}^{+}$channels, 2) Slow $\mathrm{K}^{+}$channels, 3) $\mathrm{Na}^{+}$dependent $\mathrm{K}^{+}$channel. Although the majority of research following SCI focuses on the voltage-gated ion [fast] channels due to their prominent location on myelin, overexposure to any of these can further implicate secondary injury. Normally, opening of voltage-gated ion channels creates an influx of sodium $\left(\mathrm{Na}^{+}\right)$ ions. This rapid influx depolarizes the cell, forcing the $\mathrm{Na}^{+}$channels to close, opening $\mathrm{K}^{+}$channels and $\mathrm{K}^{+}$is effluxed. Demyelination exposes these $\mathrm{K}^{+}$channels, increasing their activity which in turn shunts $\mathrm{Na}^{+}$, inhibiting action potential generation. The inhibition interrupts signal conduction, which further damages cells and releases excitotoxins [6,7]. 


\section{Sodium}

Sodium channels are found within neurons, myocytes, and glial cells. Often membrane gradiences of other channels are dependent on $\mathrm{Na}^{+}$ions. Similar to $\mathrm{K}^{+}$channels, $\mathrm{Na}^{+}$channels are essential in generating action potentials and are affected by SCI. Again, damage to the myelin sheaths disrupts the cellular homeostasis of $\mathrm{Na}^{+}$and $\mathrm{K}^{+}$, consequently inhibiting action potential generation and creating axonal conduction dysfunction. Also due to demyelination is activation of the $\mathrm{Na}^{+}-$ $\mathrm{Ca}^{2+}$ exchanger, which increases intracellular levels of $\mathrm{Ca}^{2+}$, leading to a myriad of events that also induce secondary damage. Disregulation of $\mathrm{Na}^{+}$and $\mathrm{K}^{+}$homeostasis has also been found to increase glutamate release which contributes to excitotoxicity post SCI. Finally, because of their location on neurons, over-expression of $\mathrm{Na}^{+}$channels has also been linked to neuronal excitability. This correlates $\mathrm{Na}^{+}$channel malfunction to central neuropathic pain, which can be present after injury to the CNS $[6,7]$.

\section{Magnesium}

Following SCI, there is a decrease in intracellular magnesium, which serves as a neuroprotector by blocking NMDA receptors. Thus, decreased magnesium can contribute to the increase in intracellular calcium, and which indirectly contributes to calciummediated secondary injury. Studies have shown that magnesium administration may be given as late as 8 hours post-SCI and show beneficial effects. Although a dose of $600 \mathrm{mg} / \mathrm{kg}$ of magnesium sulfate has been shown to be optimal in mice, this does is outside tolerable human limits. Thus, for human treatment, lower doses of magnesium when combined with polyethylene glycol (PEG) have also been found to increase tissue paring at the primary injury site and improvements in BBB at 6 weeks post-injury. With multiple ion concentrations being changed following SCI, it is important to explore therapeutic means to regain homeostasis, and magnesium supplementation may be one way to do this[6,7].

\section{Calcium-Mediated Secondary Injury}

Another form of glutamate excitotoxicity occurs through the activation of $\mathrm{Ca}^{2+}$ systems. The continuous axonal depolarization from increased glutamate eventually leads to a massive increase in intracellular $\mathrm{Ca}^{2+}$ via voltage-dependent $\mathrm{Ca}^{2+}$ channels and opening of NMDA receptor channels. Eventually, selfdestructive calcium-dependent enzymes are activated which also trigger cell death. Once intracellular levels of $\mathrm{Ca}^{2+}$ increase, nitric oxide synthase occurs, resulting in increased activation of poly (ADP ribose) polymerase-1 (PARP1). PARP1 is an enzyme associated with cell differentiation and proliferation, and helps regulate DNA damage during natural occurring cell death. Additionally, there is an increase in apoptosis inducing factor (AIF) translocation to the cell nucleus, damaging the DNA damage.

Increases in intracellular $\mathrm{Ca}^{2+}$ levels leads to impaired cellular respiration, which is a function of the mitochondria. Additionally, the increased $\mathrm{Ca}^{2+}$ levels stimulate calciumdependent proteases (break down peptide bonds holding proteins together) and lipases (break down fats), including calpains, phospholipase A2, lipoxygenase, and cyclooxygenase. The activation of lipase, lipoxygenase, and cyclooxygenase leads to the increased conversion of arachidonic acid into thromboxanes, prostaglandins, and leukotriene $[6,7]$.

\section{Catecholamines}

Epinephrine (Epi) and norepinephrine (Nor) are catecholamines produced by chromaffin cells of the adrenal medulla located in the adrenal cortex. While Epi is specific to the adrenal medulla, Nor is also produced by postganglionic fibers of the sympathetic nervous system. Catecholamine release is regulated by the sympathetic nervous system; when activated, increased concentrations of catecholamines are released into circulation. The sympathetic nervous system has a powerful influence on both cardiovascular and pulmonary functions, as well as metabolic processes. Spinal cord injury results in increased activation of the sympathetic 
nervous system (SNS), causing increases in plasma concentrations of the catecholamines epinephrine and norepinephrine (NE). The alterations in catecholamine levels correspond to changes in heart rate, stroke volume, blood pressure, and metabolism found post-SCI.

Within 30 minutes of spinal cord injury, NE levels double and continues to increase throughout the first hour to four times the original level. A steady decrease occurs over the next few hours until baseline values are reached, typically around four hours postinjury. Increases in NE levels have been linked to increased hemorrhage and necrosis levels in the central gray matter of the spinal cord. These levels of NE are toxic and can induce vasospasms of the vascular system which further decreases spinal cord blood perfusion and leads to cell necrosis and vascular rupture. Catecholamines have been found to possess strong influences on the immune system. This relationship is made possible due to the presence of $\beta \&$ a-adrenergic receptors found on the plasma membranes of neutrophils, macrophages, $\mathrm{T}$ and $\mathrm{B}$ lymphocytes and natural killer cells. Also, noradrenergic nerves have been found to innervate immune cell storage tissues, thus stimulating their release into circulation. Therefore, catecholamine release corresponds to a large increase in leukocytes and lymphocytes [6,7].

While neutrophils numbers will increase, catecholamines have also been found to inhibit their functions through decreasing their ability to phagocytose foreign material or release lysosomal enzymes. Catecholamines also inhibit $\mathrm{T}$ helper cell's release of antigens through binding to $\beta 2$-adrenoreceptors on the cell membranes. Catecholamines have also been shown to inhibit pro-inflammatory cytokines such as TNF- $\alpha$, IL- $1 \beta$ and IL-12 from dendritic cells, monocytes and blood cells, while also facilitating the production of the anti-inflammatory IL-10 cytokine. Catecholamines have been found to lead to an overall decrease of immune cells after prolonged or repeated exposure, specifically NK and lymphocytes [6,7].

\section{Cortisol}

Individuals with spinal cord injuries, both paraplegics and tetraplegics, were found to have relatively higher levels of cortisol as compared to non-injured age and gender matched controls. Cortisol inhibits many proinflammatory mediators such as cytokines (IL$1,2,3,4,6$, and 8 , TNF- $\alpha$, and IFN- $\gamma$ ), phospholipids, and proteases. Other antiinflammatory actions of cortisol include promoting antigen uptake by phagocytes, decreasing proliferation and increasing apoptosis in eosinophils and T-lymphocytes. Through these interactions, it becomes apparent cortisol leads to an overall decrease in immune system function [6,7].

\section{Apoptosis and Necrosis}

Secondary injury consists of two mechanisms of cell death which assist in mediating further spreading of the injury. The first being necrosis, followed by apoptosis. Necrosis is often described as premature cell death amongst living tissue. It is often characterized as swelling of the cell, resulting in interruptions in the cell's homeostasis, leading to cell lysis. This event is caused by external factors, such as infection or trauma. The second mechanism, apoptosis (often referred to as programmed cell death), is a natural occurring cell death within the body. This is a domino-like effect of "cell suicide" in an organized, sequential fashion involving a myriad of various biological events. Although the two are in contrast to one another, necrosis and apoptosis occur simultaneously during the early stage of secondary injury. In later stages, apoptosis is present independently of necrosis. Necrosis kills cells via cell lysis by disrupting its homeostasis, resulting in swelling and later death of the cell. This then causes the extracellular environment to be exposed to the intracellular content, creating an inflammatory response [6,7].

Following SCI, apoptosis occurs among astrocytes within the epicenter, and in microglia in the surrounding gray and white matter [6,7]. 
Much research has been done studying mitochondrial dysfunction following SCI. As previously described, excitotoxicity occurs in consequence to neuronal injury post-SCI. Research has linked excitotoxicity to increased buffering of $\mathrm{Ca}^{2+}$ by the mitochondria, leading to mitochondrial dysfunction and interrupting the electron transport chain (ETC). The ETC is a process induced by the mitochondria as a means of providing energy to the cell. Here, the mitochondria produces ATP and prematurely reduces oxygen, forming reactive oxygen species (ROS). Disruptions in this process can alter the production of ATP and ROS, consequently increasing production of ROS and negatively impact mitochondrial homeostasis. These mechanisms take place within the first 24 hours following SCI [6,7].

Two forms of Heat shock proteins (HSP) are highly associated with apoptosis following SCI: HSP-27 and -70, labeled based on their overall shape. When apoptosis is initiated by stress-induced factors, HSPs step in to inhibit this process. HSP-27 is involved in the Fasligand binding mechanism of apoptosis. Within this apoptotic-mediated mechanism, HSP-27 prevents the ligand binding on the cell membrane, preventing apoptosis. HSP-70 prevents apoptosome formation by binding to Apaf-1, reducing its ability to recruit procaspase-9, thus inhibiting apoptosis.

Naturally, because of the implications apoptosis has following SCI, some research has recently been geared toward studying the involvement of miRNA. Studies have shown that an insult to the spinal cord impacts miRNA, but the effects are dependent on the roles of each. Two hundred and sixty-nine miRNAs were identified in the spinal cord, and approximately 60 were altered following SCI. Of these 60 , some have been identified as dominant players in the regulation of apoptosis $[6,7,8]$.

\section{SCI ant the imune system}

Following spinal cord insult, peripheral immune cells are recruited to the injured site by local epithelial tissues and macroglia that release mediators to initiate the acute inflammatory response. Pro-inflammatory mediators include such cytokines as: tumor necrosis factor $\alpha(\mathrm{TNF} \alpha)$, interleukin (IL)-1, IL-6 and IL-8 which are released to break down extracellular components, increases vascular permeability, and signals for leukocyte/lymphocyte recruitment to the site of injury. Peripheral immune cells are transported across the BBB by the adhesion molecules intercellular adhesion molecule-1 (ICAM-1) and vascular cell adhesion molecule-1 (VCAM-1) being enhanced on the epithelial and other cells, allowing leukocyte and lymphocyte adhesion via a receptor-ligand pair with lymphocyte function-associated molecule-1 (LFA-1). The integrity of the BBB is further impaired through degradation of extracellular matrix and proteins by mediators such as matrix metalloproteinases (MMPs) $[6,7]$.

\section{References}

1. Charles Aidemise Oyinbo - Secondary injury mechanisms in traumatic spinal cord injury: a nugget of this multiply cascade, Acta Neurobiol Exp 2011, 71: 281-299;

2. Tasneem Zahir, Hiroshi Nomura, Xiao Dong Guo, Howard Kim, Charles Tator, Cindi Morshead, Molly Shoichet - Bioengineering Neural Stem / Progenitor Cell-Coated Tubes for Spinal Cord Injury Repair, Cell Transplantation, Vol. 17 (2008), pp. 245-254;

3. Aajay S. Yekkirala, David P. Roberson, Bruce P. Bean, Clifford J. Woolf - Breaking barriers to novel analgesic drug development, doi: 10.1038/nrd.2017.87, Nature Reviews / Drug Discovery, Vol. 16 (2017): 545564 ;

4. Alexandre Fogaça Cristante, Tarcisio Eloy Pessoa, de Barros Filho, Raphael Martus Marcon, Olavo Biraghi Letaif, Ivan Dias da Rocha - Therapeutic approaches for spinal cord injury, DOI: 10.6061/clinics2012(10)16, Clinics 2012; 67(10):12191224;

5. Yang D. Teng, Erin B. Lavik, Xianlu Qu, Kook I.Park, Jitka Ourednik, David Zurakowski, Robert Langer, Evan Y. Snyder - Functional recovery following traumatic spinal cord injury mediated by a unique polymer scaffold seeded with neural stem cells, PNAS, March 5, 2002, Vol. 99, no. 5, 3024-3029;

6. http://pt851.wikidot.com/spinal-cord-injury-cellbiology

7. Randall J. Dumont, David O. Okonkwo, Subodh Verma, R. John Hurlbert, Paul T. Boulos, Dilantha B. Ellegala, Aaron S. Dumont - Acute Spinal Cord Injury, Part I: Pathophysiologic Mechanisms, Clinical Neuropharmacology, 2001, Vol. 24, No. 5, pp. 254-264; 8. Elisa M. York \& Audrey Petit \& A. Jane Roskams Epigenetics of Neural Repair Following Spinal Cord Injury, Neurotherapeutics (2013) 10:757-770 